\title{
Mismatch Repair Protein Proficient
}

National Cancer Institute

\section{Source}

National Cancer Institute. Mismatch Repair Protein Proficient. NCI Thesaurus. Code C131458.

Indicates that normal concentrations of unmutated mismatch repair proteins have been detected in a sample. 